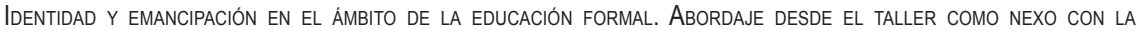
educación Popular. Páginas 99-118 en Revista de la Escuela de Ciencias de la Educación, año 12, nRo. 11, vol.1, ENERO A JUNIO DE 2016. ISSN 1851-6297. ISSN EN LINEA 2362-3349.

\title{
IDENTIDAD Y EMANCIPACIÓN EN EL ÁMBITO DE LA EDUCACIÓN FORMAL. ABORDAJE DESDE EL TALLER COMO NEXO CON LA EDUCACIÓN POPULAR
}

\author{
Natalia Jorgelina Forlini* \\ Universidad Nacional de Rosario, Argentina. \\ nataliaforlini@ hotmail.com
}

Recibido: 15/03/2016 Aceptado: 21/04/2016

\section{Resumen}

Las reflexiones que aquí se esbozan son producto del trabajo realizado desde el proyecto de investigación "Apropiación de conocimiento y cambio subjetivo. Análisis del taller como dispositivo de intervención en educación"; perteneciente a la Facultad de Psicología de la Universidad Nacional de Rosario. Aunque también surgen de las dificultades cotidianas que se observaron en las prácticas escolares y los formatos que fueron tomando el trabajo diario con éstas. Se trata de articular diferentes problemáticas frecuentes en los ámbitos de educación, poniendo en el centro del debate a los obstáculos, para visualizarlos no como escollos limitantes, sino como emergentes posibilitadores de una educación popular y emancipadora.

En tal sentido, se presenta el ensamble de las sobredeterminaciones sociales a las problemáticas que plantea el vínculo educador educando en la dinámica de taller y en los procesos del enseñar y del aprender que allí se producen. Se centra el interés en aquello que resulta ineludible pensar, es decir, en cómo se configura (o no) el lazo entre quien enseña y quien aprende, sin olvidar la necesaria transmisión de conocimientos y saberes, ni el impacto que tiene este vínculo sobre la configuración de la identidad, la autoestima y la afectividad.

* Doctora en Ciencias de la Educación por la Facultad de Humanidades y Ciencias de la Educación de la Universidad Nacional de La Plata. Becaria de CONICET: Beca Interna de Posgrado Tipo I y Tipo II. Licenciada y Profesora en Ciencias de la Educación. Profesora adjunta en la cátedra Área del Sujeto de la carrera de Ciencias de la Educación, Facultad de Humanidades y Artes UNR. CATEGORIA V. Miembro (Integrante activo) del equipo perteneciente a la Red en Educación para el Desarrollo Sustentable, Universidad Nacional de Rosario, adherida a la Red "Educación para el Desarrollo Sustentable" Reorienting Teacher to Address Sustainability, UNESCO - UNITWIN, Universidad de York Canadá. 
Revista de la Escuela de Ciencias de la Educación, año 12, nRo. 11, vol. 1, enero a Junio de 2016. Páginas 99-118. ISSN 1851-6297. ISSN EN LINEA 2362-3349. IDENTIDAD Y EMANCIPACIÓN EN EL ÁMBITO DE LA EDUCACIÓN FORMAL. ABORDAJE

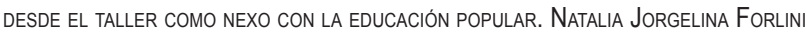

\title{
Palabras clave:
}

Identidad - Emancipación - Educación Popular - Taller - Educación Formal.

\begin{abstract}
The reflections outlined here are the result of work done from the research project "Ownership of knowledge and subjective change. Analysis workshop as interventional device in education"; belonging to the Faculty of Psychology at the National University of Rosario. But also they arise from the everyday difficulties observed in school practices and formats was taking the daily work with these. This is articulate different frequent problems in the fields of education, placing at the center of the debate obstacles to visualize not as limiting pitfalls, but as emerging enablers of a popular and emancipatory education.

In this regard, the assembly of the social to the problems posed by the educator in the dynamic link educating workshop and in the processes of teaching and learning that take place there overdeterminations occurs. interest in what is unavoidable to think, that is, on how it is configured (or not) the link between who teaches and who learns, without forgetting the necessary transfer of knowledge and know-how, nor the impact of this relationship on focus configuration of identity, self-esteem and affection.
\end{abstract}

\section{Keywords:}

Identity - Emancipation - Popular Education - Workshop - Formal Education.

\section{Introducción}

Sobre el tema que convoca este escrito, la identidad y la emancipación en la educación formal y su abordaje desde la propuesta de talleres, siendo estos el nexo con la educación popular, implica problematizar el acontecer escolar y su estructuración cotidiana. Inicialmente, cabe señalar qué es lo que entendemos por "problemática". Se trata de la producción de una ruptura en la naturalización del devenir de algo que aparece como dado, algo que se ha establecido como obvio y que, por lo tanto, no se cuestiona. Esta noción cobra especial relevancia al abordar cierta tendencia a la naturalización del campo social, que hace que un fenómeno deje de percibirse como causado para entrar a formar parte de lo cotidiano, lo habitual.

De este modo, las percepciones del mundo social, los códigos, las interpretaciones, las visiones valorativas y estandarizadas, por ser comunes a todos los sujetos de un orden social ya establecido, llegan a imponerse con toda la apariencia del sentido común. Como resultado de este proceso, son tomadas sin controversias, sin que puedan ser concebidas a la luz de su carácter relativo; así, dejan al sujeto en un estado de pasividad que conduce a la alienación en la vida cotidiana. 
Revista de la Escuela de Ciencias de la Educación, año 12, nRo. 11, vol. 1, enero a Junio de 2016. Páginas 99-118. ISSN 1851-6297. ISSN EN LÍNEA 2362-3349. IDENTIDAD Y EMANCIPACIÓN EN EL ÁMBITO DE LA EDUCACIÓN FORMAL. ABORDAJE DESDE EL talleR COMO NEXo CON LA EdUCACIÓn POPULAR. Natalia Jorgelina ForlinI

Entendemos que la vida cotidiana "es la forma de desenvolvimiento que adquiere día tras día nuestra historia individual, (...) nos muestra un mundo subjetivo (...) pero a la vez ese mundo es intersubjetivo, social y compartido, porque es un mundo que vivimos con otros" (Quiroga, 2007:12); de allí su carácter autoevidente, es decir, la cualidad de presentarse como lo que no tiene sentido cuestionar. Su desenvolvimiento se enmarca en una familiaridad acrítica que, desde la apelación a un presunto orden natural, encubre y distorsiona el carácter social de las manifestaciones concretas antes mencionadas.

Se hace necesario, por lo tanto, iniciar un proceso de desnaturalización del conocimiento sobre el campo psico-social educativo y los vínculos humanos que allí se producen. Al respecto, partiendo de una posición dialéctica, se entiende a la práctica como tensionante de la teoría y como habilitante del surgimiento de un nuevo conocimiento; este será, en consecuencia, una síntesis de la actividad práctica nunca acabada. Sepropone así, una praxis, aun cuando no permanece ajena a los múltiples factores condicionantes que sobre definen el movimiento de la producción cognoscitiva.

De una escuela ciega, sorda y muda a una escuela pública, laica, popular y emancipadora. Sobre los diferentes discursos de inclusión

De acuerdo con la línea de análisis propuesta previamente, los interrogantes se presentan en relación a cómo se expresan algunos conceptos dentro de las prácticas habitualesen una institución educativa, y considerar las herramientas con que cuentan las escuelas para operar en el día a día. En tal sentido, procuraremos clarificar en qué esferas terminan ubicados el educador y el educando en el diseño de la cotidianidad escolar, ya que es el vínculo entre la identidad y la transmisión de la cultura en la escuela lo que convoca a reflexionar sobre dicha cotidianidad en términos de problemática.

Algunos teóricos (Meyer, Ramírez, 2002) proponen como política de Estado la inclusión de la educación en el sistema globalizado de transmisión de conocimientos. ¿Qué significa esto? Básicamente, institucionalizar la educación a escala mundial con un sistema curricular que involucre a todos los Estadosnación, y de este modo, universalizarla. Veamos una cita:

Se considera la educación como parte de un modelo universalista y global (...), como un modelo cultural de desarrollo y justicia, (...) como una empresa mundial, universal y universalista en cuanto a sus aspiraciones y, en cierta medida, en cuanto a sus resultados (p.94).

Al respecto argumentan:

la educación, pues, no se halla muy adaptada a las idiosincrasias infinitamente variadas de la realidad social, sino que se homogeiniza 
Revista de la Escuela de Ciencias de la Educación, año 12, nRo. 11, vol. 1, enero a Junio de 2016. Páginas 99-118. ISSN 1851-6297. ISSN EN LINEA 2362-3349. IDENTIDAD Y EMANCIPACIÓN EN EL ÁMBITO DE LA EDUCACIÓN FORMAL. ABORDAJE

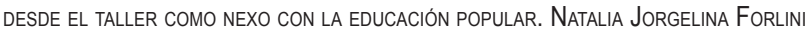

alrededor de objetivos o proyectos de desarrollos comunes a todos y de ideas tecnológicas igualmente comunes, acerca de cómo alcanzar esos objetivos (...). La educación se halla estandarizada porque forma parte de un modelo general del moderno Estado-nación (...). La elaboración de la educación como un modelo relativamente específico en sí mismo intensifica mucho la estandarización global (p. 95).

Precisamente, estos autores plantean que es el Estado el que se integra a las nuevas necesidades del mundo globalizado, sin perder de vista que tales necesidades se relacionan con la forma en que la educación —a modo de engranaje- se integra, a su vez, a este nuevo modelo de acumulación. En cierto modo, se termina formalizando aquello que, muchas veces encubiertamente, algunos organismos internacionales planificaron para los distintos países de América Latina.

Cabe señalar la forma en que se hace referencia al valor local de cada individuo o región desde esta perspectiva teórica, al abordarse como rasgos especiales de carácter nacional o estilo cultural aquellas peculiaridades que son propias de una región de algún país:

Las naciones y los individuos pueden y, de hecho, a menudo tienen que aspirar a mostrar algún valor singular en sus estilos o historiales. Tales estilos se aplican en una gran variedad de ámbitos, desde los temas culinarios y los estilos en el vestir, hasta las representaciones artísticas y literarias respecto de las constituciones de la historia nacional y de la cultura indígena (p.105).

Lo que resulta especialmente interesante en estas citas es el lugar en que se ubican las particularidades culturales. Por una parte, son objeto del desprestigio y el descrédito en tanto saber condicionante de lo escolar; por otra parte, se las trata como un "adorno secundario" de poca o mínima participación en la definición de la configuración curricular —en sentido amplio— de la educación formal.

En resumen, el realce de lo propio aparece como un elemento que no hay que suplantar ni borrar; sin embargo, este aparente reconocimiento no resulta satisfactorio, ya que se deja de lado, en pos de priorizar los modelos y estándares globales, lo que simbólicamente representan dichas prácticas culturales para quienes las producen y reproducen.

Dentro de la propuesta con la que se está discutiendo se hace mención, entonces, aunque al modo de una "pincelada costumbrista", de las características particulares de cada región. Para ese punto de vista, la identidad implica cuestiones que refieren a una gran variedad de ámbitos, pero se la sigue abordando como si, en cambio de algo formado y transformado socialmente, se tratara de estilos de personalidad; es decir, se enfatiza lo individual. 
Revista de la Escuela de Ciencias de la Educación, año 12, nRo. 11, vol. 1, enero a Junio de 2016. Páginas 99-118. ISSN 1851-6297. ISSN EN LÍNEA 2362-3349. IDENTIDAD Y EMANCIPACIÓN EN EL ÁMBITO DE LA EDUCACIÓN FORMAL. ABORDAJE DESDE EL talleR COMO NEXo CON LA EdUCACIÓn POPULAR. Natalia Jorgelina ForlinI

Ahora bien, ante estas posiciones, resulta difícil imaginar cómo podrían las diferentes comunidades y grupos involucrar sus propias identidades en lo referido al ámbito escolar. En realidad, con la implementación de una perspectiva semejante en las organizaciones escolares, se terminaría favoreciendo un proceso de afianzamiento de la globalización de la cultura. De este modo, se busca homogeneizar, imponer ciertos cánones culturales universalmente aceptados para que sean adoptados con naturalidad por las nuevas generaciones.

Por otra parte, este proyecto educativo globalizante, que borra las diferencias con las que parten los aprendientes de diferentes sectores sociales, podría estar generando discursos falaces en torno al acceso y la permanencia de los educandos en el Sistema Educativo formal de un Estado-nación.

Estas políticas, que Ilamaremos "de estandarización de la educación mundial”, no se contradicen, a decir verdad, con el avance y el afianzamiento del nuevo orden capitalista mundial.

Es por ello que resulta necesario preguntarnos, una vez más, cómo se expresan en el Sistema Educativo Argentino estos cambios, y cómo se traducen en las prácticas educativas cotidianas. Es decir, ¿con qué herramientas contamos para operar con respecto a estas cuestiones en el día a día? En los apartados que a continuación se presentan abordamos la dinámica del taller como propuesta alternativa a la que presenta en la estandarización de la educación mundial.

\section{Identidad, emancipación y educación en clave de transmisión cultural}

Existe una gran variedad de definiciones en torno a la identidad. En este apartado se focaliza en su relación con la transmisión de la cultura y con los procesos emancipatorios en los sujetos. El concepto de identidad queda separado de aquello que hace referencia a cuestiones de orden sobrenatural o metafísico - la identidad como algo que hay que descubrir en el interior espiritual de cada individuo-, así como de determinaciones naturales con las que el individuo nace. (1)

En principio hay un acuerdo en sostener que la identidad aparece conformada a través de la acumulación histórica de elementos formados por ideas, sentimientos, prácticas simbólicas, costumbres, creencias y representaciones; todo ello produce un sedimento que da como resultado la identidad de un sujeto. Es allí, en una interacción mutua entre los sujetos, donde diferentes procesos del devenir social actuarían como "modificadores" de la identidad. La identidad, por lo tanto, no existiría como entidad estática y abstracta, sino como producto de una construcción constante; se trataría, más precisamente, de un proceso de cimentación y deconstrucción continuas. Como las capas de una cebolla, estos diferentes aspectos envuelven al yo y le otorgan, así, su identidad. Sin embargo, es esa misma acumulación de elementos la que, a su vez, permite la 
Revista de la Escuela de Ciencias de la Educación, año 12, nRo. 11, vol. 1, enero a junio de 2016. Páginas 99-118. ISSN 1851-6297. ISSN EN LINEA 2362-3349. IDENTIDAD Y EMANCIPACIÓN EN EL ÁMBITO DE LA EDUCACIÓN FORMAL. ABORDAJE

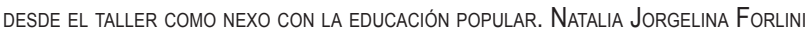

diferenciación entre unos y otros, y la posibilidad de armar una grupalidad. Por supuesto, tales procesos no se presentan aisladamente ni sin contradicciones.

A diferencia de las líneas teóricas que abordan el concepto de identidad quitándole la faz conflictiva al proceso de sedimentación, el enfoque que se aborda -y que proponemos aplicar en el siguiente análisis_ plantea que el entramado social no puede ser abordado con independencia de la interrogación histórica dirigida al contexto en el cual se origina. Asimismo, lo individual es abordado en ese encuentro con la historia social; es decir, como producto y consecuencia de la estructura social. La cultura — como proceso civilizatoriomuestra su producción plasmándose en la identidad de cada sujeto.

Finalmente se sintetiza, en la cita que a continuación se transcribe, la concepción de identidad:

(...) es el resultado de un proceso de constitución continua, durante el cual diversos elementos contradictorios no solo se unen sino que se mantienen en tensión y disputa. En este proceso hay cambios y continuidad. $Y$ se va conformando tanto en cada individuo, como en lo colectivo una totalidad de elementos que permiten a la comunidad y a cada uno de sus miembros, identificarse a la vez que diferenciarse. Estamos refiriéndonos a la continuidad histórica de una comunidad relativamente estable, que a lo largo del tiempo gesta un complejo de culturas, de lengua, de psicología, que adquiere conciencia de sí y de los otros como diferente y semejante (Racedo, 2004, p. 21).

De ahí que acordamos en que:

historicidad, unidad, continuidad, hacen a ese proceso social y subjetivo denominado identidad. (...) Ella es el soporte interno del sujeto y de las organizaciones. Desde su identidad que implica movimiento, semejanza y diferencia, el sujeto se sitúa en el presente y anticipa un futuro, sostenido y sosteniendo un proyecto que da sentido a su vida personal y a su ser social. Este proyecto será entonces su referente, su sostén. Desde el proyecto adquiere una dimensión de lo posible, una apertura y una capacidad anticipatoria con posibilidad de "planificar la esperanza (E. Pichón-Rivére) (en Quiroga, 2005, p. 29).

El concepto de identidad es diferente al concepto de subjetividad. La subjetividad —al igual que la identidad — se relaciona con los condicionantes materiales de la existencia, está moldeada por lo sociocultural y determinada por el momento histórico en el que transcurre. Es decir, aunque la subjetividad - como aquello singular del individuo — es la cualidad en la que se plasma el psiquismo, el modo histórico de producción imprime en el sujeto determinadas 
Revista de la Escuela de Ciencias de la Educación, año 12, nRo. 11, vol. 1, enero a Junio de 2016. Páginas 99-118. ISSN 1851-6297. ISSN EN LÍNEA 2362-3349. IDENTIDAD Y EMANCIPACIÓN EN EL ÁMBITO DE LA EDUCACIÓN FORMAL. ABORDAJE DESDE EL talleR COMO NEXo CON LA EdUCACIÓn POPULAR. Natalia Jorgelina ForlinI

singularidades psíquicas. De ahí que se entienda la constitución del sujeto y su subjetividad por lo sociocultural, lo histórico y libidinal.

La subjetividad se mueve como un péndulo entre la introyección de lo establecido - lo que el sujeto introyecta de la sociedad, las leyes del superyó-y su potencial de subvertir, crear, instituir algo diferente; la posibilidad del sujeto de oponerse a lo instituido opera como una adaptación activa. La identidad se entrelaza necesariamente con la subjetividad (Franco, Freire, Loreti, 2007).

Cuando se habla de formación de identidades, se hace referencia, en términos generales, a ponerle palabras a la pregunta por "quién soy"; que, a su vez, remite a una historia subjetiva, familiar y social, y a todas aquellas prácticas y relaciones que forman sujetos sociales (Quiroga, 1996).

La construcción de la identidad, por lo tanto, es un trabajo que realiza el yo - como instancia psíquica — del sujeto que, activamente, va tomando aspectos de la cultura y de lo histórico social, para dar una respuesta nunca acabada a la pregunta -ya mencionada- “¿quién soy?”; y a ella se le agrega —aunque en realidad no se consideran separadamente-, la pregunta por "qué puedo hacer". La identidad social puesta en estos términos se potencia en la esfera pública, mientras que la subjetividad pertenece a lo más íntimo y privado del sujeto; aunque, insistimos, una y otra se retroalimentan constantemente.

Desde este enfoque, se entiende por cultura lo que Josefina Racedo expresa como "el conjunto de realizaciones materiales y espirituales, producidas y materializadas por los integrantes de un pueblo, que se encuentra a sí mismo en ese hacer, (...) y solo existe por la relación organizada entre los miembros de una sociedad" (2004, p.30).

Identidad y cultura se constituyen como el anverso y el reverso de un proceso histórico a lo largo del cual una no se desarrolla sin la otra; no por ello desaparece la lucha entre ambas — de producción y reproducción-, que las mantiene en una situación de tirantez y tracción persistente. De ahí que Josefina Racedo entienda que la identidad es un terreno de lucha donde las clases dominantes buscan imponer un diseño afín a sus objetivos.

En el revés de la trama social se asienta, entonces, la formación de las identidades de una época; de esta manera, es posible separar el problema de la identidad de cuestiones que hacen a un orden natural, para ubicarla, en cambio, en el contexto de un proceso histórico-social.

En estas expresiones se observa que la configuración de la identidad se plasma como una síntesis. Síntesis que, por un lado, muestra las características propias del sujeto, aquello que lo singulariza; y por otro, ofrece una configuración identitaria, envuelta y estructurada por la historia social —como un claro producto de esa tensa relación que establece el sujeto con el contexto histórico en el cual se desarrolla-. En otras palabras, sepuede distinguir y relacionar la identidad con lo estrictamente histórico y social de un sujeto. 
Revista de la Escuela de Ciencias de la Educación, año 12, nRo. 11, vol. 1, enero a Junio de 2016. Páginas 99-118. ISSN 1851-6297. ISSN EN LINEA 2362-3349. IDENTIDAD Y EMANCIPACIÓN EN EL ÁMBITO DE LA EDUCACIÓN FORMAL. ABORDAJE

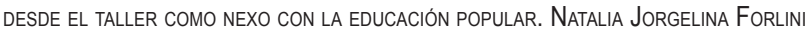

Junto a lo expuesto, se considera que la configuración de la identidad no encierra solo una trama de problemáticas intrínsecas propias del sitio que se ocupa en una época $-y$ del recorrido singular que se realiza-, sino que existen determinaciones por fuera del sujeto, hasta muy sutiles - extrínsecas, condicionadas estructuralmente-; sin que, muchas veces, este fenómeno pueda ser considerado por los propios sujetos de forma consciente como un acontecer restrictivo propio de las identidades. En relación a ello, pensamos en el "peso del legado", como si algo del orden del legado, de una herencia cultural -y del compromiso por haberla recibido- se estuviera poniendo en juego; es esto a lo que Piera Aulagnier llamó "contrato narcisista":

El contrato narcisista se instaura como un pacto de intercambio: El grupo garantiza la transferencia sobre el nuevo miembro del reconocimiento (...), el nuevo miembro se compromete (...) a repetir el mismo fragmento de discurso, (...) que repita los enunciados, garantizando así, la permanencia del grupo. El niño demandará que se le ofrezca un modelo ideal (...) que le permita conservar la ilusión de una persistencia atemporal sobre el conjunto $y,(\ldots)$ que según supone, sus sucesores retomarán y preservarán (1997, pp.163-164).

Partiendo de este señalamiento, se aprecia que la identidad se encuentra integrada por la contradicción existente entre lo individual y lo colectivo; como dos niveles de determinación que se cruzan y tensionan entre sí. Desde lo individual, entendido como lo histórico singular —como ya dijimos-, se hace referencia a los procesos de constitución psíquica de un sujeto particular -apuntando tanto a los procesos identificatorios como a la construcción de ideales-; y desde lo social colectivo, se apela al escenario concreto del desarrollo de la existencia, con su correlato en la producción y reproducción de formatos sociales. Al respecto, Norbert Elias expresa:

Se usan habitualmente los conceptos 'Individuo' y 'sociedad', como si se tratara de dos sustancias pasivas distintas. Al emplear así estas palabras, se suscita fácilmente la impresión de que aquello que denotan, no solo son objetos distintos, sino que existen absolutamente separados; en realidad son procesos que, sin duda alguna, pueden distinguirse, pero no separarse (1996, p.33).

Es interesante también retomar la propuesta de Racedo (2004) en relación al vínculo entre individuo y sociedad, cuando propone entender ese vínculo como la conformación de una unidad de opuestos en el interior de la identidad. Allí interviene un juego de disputa dialéctica entre, podríamos decir, un polo al que denomina "la identidad asignada", y otro polo designado como "la identidad emancipada". La primera se encuentra especificada por los valores y discursos de la clase dominante y, en este sentido, señalada desde el deber ser y el ser 
Revista de la Escuela de Ciencias de la Educación, año 12, nRo. 11, vol. 1, enero a Junio de 2016. Páginas 99-118. ISSN 1851-6297. ISSN EN LÍNEA 2362-3349. IDENTIDAD Y EMANCIPACIÓN EN EL ÁMBITO DE LA EDUCACIÓN FORMAL. ABORDAJE DESDE EL talleR COMO NEXo CON LA EdUCACIÓn POPULAR. Natalia Jorgelina ForlinI

nacional; la segunda, en cambio, se construyó y se construye desde la concientización del lugar que se ocupa en la estructura del sistema de dominación, pero sin aceptar dicha posición.

Esta autora, habla de una dialéctica que actúa en el núcleo de la configuración de la identidad. Allí se registran dos aspectos: uno donde se juegan elementos de los sectores dominantes y otro en el que se concentran los elementos de resistencia. De la lucha y tensión entre lo asignado y lo emancipatorio resulta la síntesis que se denomina "identidad". Desde ahí se otorga significación al mundo y se lo resignifica; porque desde esa posición se construye el esquema referencial de sentidos del mundo del sujeto, aquello que le da razón de ser a la propia identidad.

\section{La identidad de los sectores populares en las prácticas educativas}

Emergen preguntas tales como: ¿puede la escuela sostener y ofrecer un proyecto cuando las identidades se están configurando actualmente bajo el predominio de la incertidumbre?; y ¿cómo podrá sostenerse un proyecto integral a futuro en este presente que reúne la fragmentación y la fluidez de los vínculos como elementos instalados en la vida cotidiana? El concepto de Aulagnier, denominado "proyecto identificatorio", aporta una nueva arista de complejidad. Según la autora, este es "el soporte de las identificaciones que (...) el sujeto debe encontrar en el discurso de su grupo de pertenencia, referencias que le permitan proyectarse hacia un futuro" (1997, p. 159).

Lo que entendemos subyace en los planteos de la propuesta universalista de educación -y que los distancia más aún de la nuestra- está relacionado con una concepción diferente de lo que significa la educación en clave de transmisión de la cultura. La idea de transmisión de la cultura es proyectada en términos universalistas y homogeneizadores.

Si bien podría tomarse como un derecho global en relación a la educación, no puede pensarse en la mundialización como el mero objetivo de igualar y ofrecer todo a todos. En un aspecto, es cierto, hace referencia al derecho inalienable de todo sujeto de derecho a recibir educación; lo que no se plantea es en qué términos será ofrecida, es decir, cómo será enseñada, aprendida y por lo tanto posibilitadora de la emancipación del educando y su colectivo social.

Creemos por último que, dentro de esta matriz, pensar una educación universalista esconde una idea de educación que se limita a impartir conocimientos que pueden ser asimilados y aprendidos acríticamente; es decir, recepcionados pasivamente y sin cuestionamientos. 
Revista de la Escuela de Ciencias de la Educación, año 12, nRo. 11, vol. 1, enero a Junio de 2016. Páginas 99-118. ISSN 1851-6297. ISSN EN LINEA 2362-3349. IDENTIDAD Y EMANCIPACIÓN EN EL ÁMBITO DE LA EDUCACIÓN FORMAL. ABORDAJE DESDE EL talleR COMO NEXO CON LA EdUCACIÓN POPULAR. NATALIA JORgelina ForlinI

\section{La educación emancipadora frente la concepción de educación globalizadora}

Dentro de lo que se viene planteando, la propuesta de universalizar la educación deja entrever una concepción de educación diferente a la propuesta que se sostiene en este texto.

Como primera diferencia, puede mencionarse que dicha posición universalista pone el acento en lo propio, en aquello que es característico y de orden local, pero sólo como una vía de adhesión al proyecto global, y no como punto de partida ni plataforma desde donde proyectar o planificar. Tampoco es considerado como determinante de la enseñanza y del aprendizaje. Se considera, por lo tanto, que se encuadra dentro de aquellas concepciones que plantean a estos ámbitos -el de la enseñanza y el del aprendizaje- como dos actos que se dan en tiempos separados uno del otro. Desde esta postura, podría haber enseñanza, pero ello no garantiza el aprendizaje. Es decir, que se produzca el acto de enseñanza no es condición para que el alumno haya aprendido efectivamente.

De allí surge toda una discusión acerca de la unión gramatical de la enseñanza y aprendizaje; se cambia la " $y$ " por un "guión" a fin de indicar que se está hablando de dos procesos diferentes: enseñanza-aprendizaje. Se busca, de esta manera, romper con el esquema de la psicología conductista que plantea que a determinada enseñanza se debe determinado aprendizaje (esquema montado sobre el binomio "estímulo/respuesta"). Sin embargo, no hace más que afianzar la separación de estas dos instancias reforzando la idea de la desvinculación y despersonalización subjetiva de ambas. Por supuesto que no todo lo que se enseña es aprendido; como también es cierto que muchos actos de enseñanza se aprehenden en un tiempo diferente y hasta intangible.

La enseñanza y el aprendizaje, desde la perspectiva emancipadora, constituyen dos objetos de estudio diferenciados entre sí, aunque uno implica necesariamente al otro. Al referirse a sujetos que enseñan, se habla de la posición de un educador. Éste es el encargado de sostener la socialización de la vida escolar y los aprendizajes que allí se realizan para intervenir cognitivamente y propiciar la puesta en marcha del educando y del grupo de aprendizaje.

Tal posicionamiento resulta necesario para lograr la construcción de saberes por parte de los educandos a partir del conocimiento que proveen las ciencias. Es decir, el educador debe auspiciar de prótesis, de puente entre el mundo conocido o socio familiar y el escolar; entre lo que los educandos saben y deben aprender; entre las dificultades y las posibilidades; en definitiva, entre lo que deben y desean.

En cuanto al sujeto que aprende, este posicionamiento lo ubica, subjetivamente en el espacio grupal áulico, desde otra perspectiva. La diversidad de representaciones se potencia y fortalece, se abre a múltiples sentidos. El pensamiento y la identidad se enriquecen. "El encuadre grupal que supone 
Revista de la Escuela de Ciencias de la Educación, año 12, nRo. 11, vol. 1, enero a Junio de 2016. Páginas 99-118. ISSN 1851-6297. ISSN EN LÍNEA 2362-3349. IDENTIDAD Y EMANCIPACIÓN EN EL ÁMBITO DE LA EDUCACIÓN FORMAL. ABORDAJE DESDE EL talleR COMO NEXo CON LA EdUCACIÓn POPULAR. Natalia Jorgelina ForlinI

la presencia de otros actuará en ocasiones, en cada uno de ellos, como una "máquina" de acrecentar la creación de pensamientos" (Schlemenson, 1996 p.27). Parafraseando a la autora citada, puede agregarse que el grupo de pares, como referentes directo de las contradicciones de clase del grupo de referencia, posibilita el movimiento y la afirmación de la identidad del educando.

Al pensar el enseñar y el aprender en términos de una educación emancipatoria, se habla de enseñaje (Bleger, 1977). Se refiere, en sentido amplio, a la transmisión de la cultura como un posicionamiento dialéctico que los implica a ambos.Involucra, así, al sujeto en posición de enseñar, pero también incluye activamente a quien aprende. Enseñanza y aprendizaje son procesos bien delimitados pero a la vez, inseparables, integrantes de un transcurso único en permanente movimiento. Esta concepción se opone a aquellas que difunden que puede haber enseñanza, pero que puede no darse el aprendizaje. Se trata de concepciones más relacionadas con la instrucción y con pensar la educación como un adiestramiento y acumulación de información que en nada se relaciona con la transmisión, en última instancia, de la cultura.

Para que este tipo de "encuentro dialéctico" pueda producirse, tienen que tomarse críticamente las matrices pedagógicas y buscar su rematrización (Quiroga, 2012). La novedosa estructuración de los vértices de la tríada, desde un espacio de fraternidad, comunitario, de producción colectiva, surge como aspecto central de la transformación del mundo escolar. Las prácticas que emergen en la escuela son inéditas; son el producto de la actividad creadora propia en todos los sujetos. Se habla entonces de distintos sujetos del aprendizaje.

Se busca que los sujetos involucrados, tanto docentes como alumnos, se vuelvan protagonistas laboriosos de su educación, a modo de artesanos, desde el enseñaje. El enseñar y el aprender como dos polos donde uno va modificando al otro constantemente; en este sentido, la enseñanza no puede desentenderse de los procesos de aprendizaje, y estos marcarán los límites y posibilidades de la enseñanza, quien a su vez insistirá en traccionarlos. En esta dinámica, se los involucra, como intérpretes valiosos y activos, tanto a los docentes como a los alumnos.

La propuesta de enseñaje, sin embargo, solo será posible en la medida que podamos redimensionar el rol del sujeto que enseña y del sujeto que aprende; y de lo que se enseña y lo que se aprende. En síntesis, reconceptualizar el quién, el cómo, el qué y el para qué de la transmisión de la cultura.

Ambos sujetos poseen conocimientos e intereses que se diferencian de la función y rol que ejercen en el espacio escolar. En este sentido repensamos y parafraseamos a Freire (1986) cuando expresa que nadie educa a nadie, sino que los hombres en comunión se educan mediatizados por el mundo.

¿Qué sentidos pueden inferirse de esta frase? Será acaso que el educando vaya a enseñar al docente el conocimiento socialmente legitimado por el curriculum oficial. En un primer acercamiento, puede ser interpretada asumiendo, 
Revista de la Escuela de Ciencias de la Educación, año 12, nRo. 11, vol. 1, enero a junio de 2016. Páginas 99-118. ISSN 1851-6297. ISSN EN LINEA 2362-3349. IDENTIDAD Y EMANCIPACIÓN EN EL ÁMBITO DE LA EDUCACIÓN FORMAL. ABORDAJE

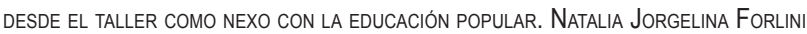

que el educando ofrecerá en ese vínculo el saber que posee de su identidad y su cultura, de su legado, de los códigos de su comunidad. Entonces, en esa famosa tríada didáctica entre el docente el alumno y el conocimiento, no hay unos arriba de otros, sino que los elementos, dentro de su propia dinámica, se complementan entre sí, se tensionan y hasta luchan por romper concepciones del status quo.

Así se obtiene que, de una tríada en donde en un momento histórico se pensó al docente por sobre el alumno (corriente tradicional), se pasó a otra, en la cual el alumno se convirtió en el eje principal (corriente escolanovista). En ambas tríadas no se interroga la estructura del sistema escolar. Las modificaciones que se propusieron, desde la corriente pedagógica de la escuela nueva, intentaron "modernizar" y/o reformar la educación tradicional sin cuestionar la raíz política de la alienación identitaria que se producía en docentes y alumnos. En la propuesta de educación emancipadora, que adhiere a la concepción de enseñaje, ambos — docente y alumno- se incluyen mutuamente, en tensión y lucha, junto al grupo de pares; y lo que circula en ese vínculo tridimensional es el conocimiento. Este último ya no se ubica en un vértice, más bien circula en permanente intercambio y construcción política; porque los vértices, reiteramos, son el educador, el educando y el grupo de pares. (2)

El gráfico siguiente resume lo que se está planteando.

\section{Tríada didáctica tradicional}

\section{Docente}

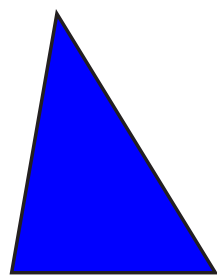


Revista de la Escuela de Ciencias de la Educación, año 12, nRo. 11, vol. 1, enero a Junio de 2016. Páginas 99-118. ISSN 1851-6297. ISSN EN LÍNEA 2362-3349. IDENTIDAD Y EMANCIPACIÓN EN EL ÁMBITO DE LA EDUCACIÓN FORMAL. ABORDAJE DESDE EL talleR COMO NEXo CON LA EdUCACIÓn POPULAR. Natalia Jorgelina ForlinI

\section{Tríada didáctica alternativa/emancipadora}

\section{Grupo de pares}

\section{Conocimiento}

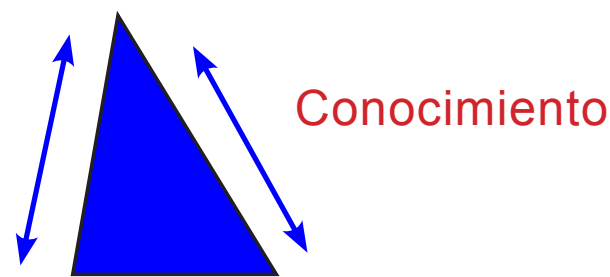

\section{Educador}

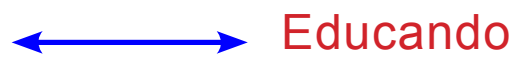

\section{Conocimiento}

Pensar sujetos y objetos en la escuela desde una perspectiva crítica y emancipadora involucra directamente la Educación Popular como aquella pedagogía que lucha contra la opresión de los sectores populares desde una perspectiva anticapitalista. Pedagogía de la resistencia a la dominación del pensamiento acrítico: educación emancipadora.

Tiene como principal objetivo la creación colectiva del conocimiento, es una pedagogía contra la alienación de la vida cotidiana. Por eso entiende de otra manera la educación, a saber, desde el empoderamiento del sujeto y de su grupo de pertenencia. En consecuencia, privilegia la perspectiva activa del sujeto, que toma su poder colectivo para apropiarse de los espacios institucionales, siendo partícipe de la propia educación y formación como sujeto social.

Desde esta concepción, la educación popular y emancipadora que se está proponiendo puede viabilizarse como un dispositivo dentro de la educación formal, ya que:

- no es una práctica para tapar agujeros del sistema;

- no es asistencialismo como cultura de la sobrevivencia;

- es una práctica social para pensar la realidad y poder transformarla;

- busca construir procesos políticos y pedagógicos colectivos;

- puede aportar a buscar la grieta en el sentido común homogeneizador, en el pensamiento único; y

- propone darle un lugar al saber popular en las escuelas, construyéndolo desde un espacio diferente. 
Revista de la Escuela de Ciencias de la Educación, año 12, nRo. 11, vol. 1, enero a Junio de 2016. Páginas 99-118. ISSN 1851-6297. ISSN EN LINEA 2362-3349. IDENTIDAD Y EMANCIPACIÓN EN EL ÁMBITO DE LA EDUCACIÓN FORMAL. ABORDAJE DESDE EL TALLER COMO NEXO CON LA EDUCACIÓN POPULAR. NATALIA JORgELINA ForLINI

El diseño de este tipo de instrumentos intenta operar con indicadores como el trabajo en grupos, el despliegue de lo propio, el pensamiento relativamente autónomo, la curiosidad, el rol del otro semejante en el grupo y el del coordinador/docente, el cuestionamiento a los esquemas conceptuales referenciales y operativos con los cuales se interpreta la realidad. Tales conceptos se anidan en el marco teórico de la psicología social de Pichon-Rivière. (3)

Cabe señalar, por otra parte, que, en relación al trabajo grupal, presenta una serie de características que resulta oportuno enumerar:

- tiene un ordenamiento necesario.

- brinda reglas establecidas que fijan una posición acerca de la producción grupal. Estos son acuerdos básicos para el funcionamiento.

- se ponen en juego procesos de identificación-diferenciación con el otro.

- la diversidad de representaciones se potencian y fortalecen, se abren a múltiples sentidos: habilitan al cuestionamiento.

- con el fin de dar lugar a los procesos de identificación-diferenciación, el grupo habilita, desde un espacio de trabajo en taller, condiciones para la generación de redes vinculares mediante la apropiación de un lenguaje de significaciones compartidas, y propicia que cada integrante se exprese en un espacio de intercambio y confianza. En este sentido, se da notable importancia a la posibilidad de generar redes solidarias que habiliten el establecimiento de vínculos generados desde el hacer de la tarea en un espacio público como lo son las instituciones educativas.

- el diálogo se transforma en una relación horizontal entre pares que habilita la confianza entre los integrantes y, al mismo tiempo, con el coordinador/docente.

- la presencia de los otros en el espacio grupal acentúa la multiplicidad y la diferencia entre estos otros; se busca, por lo tanto, generar una dinámica que habilite a la heterogeneidad en vez de sostener la ilusión de la unidad.

- la confianza permite dar a conocer lo propio, por lo que la palabra viene a ocupar el eje central de la tríada.

- la educación emancipadora implica la experiencia de hablar no solo sobre contenidos escolares, sino sobre la "vida misma"; desde aquí alcanzamos a crear, dentro del taller, un espacio abierto y libre a los decires. El dar testimonio, es decir, tomar la palabra, es para Freire (1994) el núcleo de la relación dialógica, una forma de expresión del compromiso de los hombres - como seres históricos- con la transformación de la sociedad.

- este dispositivo, los desarrollos teóricos, el contenido del trabajo están expuestos a una permanente reflexión crítica y a la autocrítica, entendidas éstas como productoras de conocimiento y, por lo tanto, 
Revista de la Escuela de Ciencias de la Educación, año 12, nRo. 11, vol. 1, enero a Junio de 2016. Páginas 99-118. ISSN 1851-6297. ISSN EN LÍNEA 2362-3349. IDENTIDAD Y EMANCIPACIÓN EN EL ÁMBITO DE LA EDUCACIÓN FORMAL. ABORDAJE DESDE EL talleR COMO NEXo CON LA EdUCACIÓn POPULAR. Natalia Jorgelina ForlinI

habilitadoras con respecto a nuevas prácticas. A partir de las síntesis que se producen, se propone la crítica como desnaturalización de las prácticas escolares.

\section{Propuesta de planificación del aula/ taller}

A continuación se describe, a modo de ejemplo, la planificación de una clase/taller desde la perspectiva emancipadora. Fue elaborada para la formación docente de un profesorado universitario. En este caso, la materia se denomina Teorías del sujeto y del aprendizaje, y se dicta en el profesorado de Matemática de la Facultad de Ciencias Exactas Ingeniería y Agrimensura de la Universidad Nacional de Rosario. Dicha propuesta puede ser transferida, con las adaptaciones correspondientes, a los distintos niveles del sistema educativo. (4)

Para la planificación se establece una problemática desde la perspectiva conceptual planteada al inicio del artículo. Se explicitan objetivos generales, específicos y los contenidos mínimos que se pretenden enseñar y/o transmitir.

La estrategia metodológica comprende un trabajo continuo de reflexiónacción y reflexión tanto del educador como del educando.Se entiende el aprendizaje en la Universidad como una forma de apropiación de los conocimientos que implica el compromiso social bajo la concepción de "praxis" —fundamento epistemológico de cómo se produce el conocimiento, síntesis de la acción y la reflexión-.

Se trabaja en una dinámica de aula/taller que incluye tres fases en su desarrollo. La primera, es una introducción que tiene un tiempo de duración de aproximadamente treinta minutos.

En este momento el educador realiza un recorrido sintético de la bibliografía para desentrañar elementos que pudieran haber quedado pendientes y desarrollar el contenido específico. Una vez que se identifica la situación conflictiva a trabajar, se sumarían las propias consideraciones y reflexiones del docente, quien culmina con el planteo de la situación en términos de problemática. Esta primera exposición, como tesis, se pone a consideración a través de la labor que se inicia en la segunda fase. En ella, el trabajo es en problematización en grupos, donde el tiempo de duración, si bien puede ser variable, implica que el educador perciba atentamente la "saturación de la discusión", es decir, cuando esta se encuentra en un proceso de estancamiento y el análisis no logra avanzar hacia posiciones que profundicen la problematización inicial.

Se presentan además la discusión y el relevamiento bibliográfico. Los alumnos realizan una reflexión crítica de lo trabajado a través de la conformación de grupos operativos de hasta cuatro integrantes. Se proponen actividades de trabajo grupal, que permitan guiar el proceso de discusión. En este momento se pone en juego la creatividad del docente para el establecimiento de ejercita- 
Revista de la Escuela de Ciencias de la Educación, año 12, nRo. 11, vol. 1, enero a Junio de 2016. Páginas 99-118. ISSN 1851-6297. ISSN EN LINEA 2362-3349. IDENTIDAD Y EMANCIPACIÓN EN EL ÁMBITO DE LA EDUCACIÓN FORMAL. ABORDAJE

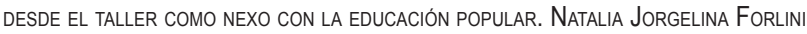

ciones que permitan y habiliten a la discusión de la bibliografía en consonancia con la problemática establecida al inicio.

La tercera fase es de Cierre o síntesis de la clase/taller. Es el tiempo para la exposición del proceso llevado a cabo y de lo producido en los grupos. Puede dividirse en dos momentos que se incluyen mutuamente. El primero que se denomina plenario colectivo: momento de síntesis de los alumnos. Cada grupo expone la producción relaciones que estableció conceptualmente. Aunque lo más importante a destacar de la exposición serán no solo las reflexiones a las que se haya arribado, sino que se dará relevancia a las discusiones efectuadas al interior del grupo y las que puedan efectuarse entre los grupos. El segundo momento serán las reflexiones del docente. La síntesis que realiza el docente se relaciona con la posibilidad de tomar lo que plantearon los alumnos para realizar una devolución de lo trabajado. Intervenir en ajustes propios para aquellas categorías que planteen confusión conceptual y/o procedimental, establecer puntos de conexión y discrepancias en lo expuesto y debatido en el taller.

Se incluye además una evaluación: como guía sobre los procesos de enseñanza y aprendizaje, es un paso necesario dentro de la praxis educativa; constituye un momento de síntesis, tanto del aprendizaje de los alumnos como del propio trabajo del docente y su planificación. Por el tipo de problemática a la que se arriba en el taller, la evaluación se inclina hacia lo cualitativo.

Criterios de evaluación del proceso de aprendizaje: se tendrán en cuenta la producción de conocimiento, el tipo de relaciones e integración del nuevo contenido que pudieron establecer los alumnos, a la vez que se considerarán el nivel de profundización de las discusiones llevadas a cabo y la forma de resolverlas (o no).

Criterios de evaluación de la práctica docente: se tendrán en cuenta, en esta reflexión sobre la propia práctica, el diseño y ejecución de la propia planificación, la utilización del material didáctico y las reflexiones a las que hayan arribado los propios alumnos sobre el tema/problema trabajado. Se agregan también aquellas apreciaciones de estos sobre el desarrollo de la práctica

\section{Conclusiones o discursos que cierran... discursos que abren}

La temática analizada implica una problemática que se presenta como un desafío inédito ante el cual nos posicionamos política e ideológicamente para el ejercicio de nuestras propias prácticas. Pero esto sólo es posible si conocemos la realidad de los sujetos con los que trabajamos. Porque el trabajo docente tiene la particularidad y, se podría agregar además, el beneficio, de que pensar la propia labor implica pensar la producción con otros sujetos.

Más que homogeneizar adaptativamente, se intenta rescatar las identidades y el legado cultural que corresponden al discurso del conjunto social de pertenencia de los educandos. Se trata de afirmar las diferencias en la integra- 
Revista de la Escuela de Ciencias de la Educación, año 12, nRo. 11, vol. 1, enero a Junio de 2016. Páginas 99-118. ISSN 1851-6297. ISSN EN LÍNEA 2362-3349. IDENTIDAD Y EMANCIPACIÓN EN EL ÁMBITO DE LA EDUCACIÓN FORMAL. ABORDAJE DESDE EL talleR COMO NEXo CON LA EdUCACIÓn POPULAR. Natalia Jorgelina ForlinI

ción. Quizá, partiendo desde allí hacia la reconstrucción de significados que hacen a lo escolar, sea posible rescatar proyectos identificatorios del colectivo. Esta búsqueda por resignificar el lugar de la transmisión intenta ir más allá de la llana propagación de cánones culturales propios de una identidad asignada y externa a los sectores populares, y finalmente, busca que dichos sectores tiendan a la edificación de una identidad emancipada.

Una propuesta de estas características configura una proposición convergente en la acción como primer puente de la actividad creadora. Se trata de partir desde la educación emancipadora para impulsar el acceso a la crítica, herramienta decisiva de cara a la pugna contra el pensamiento único. Tal posición se orienta a la prevención del fracaso escolar, a la inclusión activa y comprometida del sujeto del aprendizaje y, por último, a mejorar las condiciones del trabajo del docente. Es decir, se piensa otro escenario posible en las instituciones educativas.

Si bien ya se están realizando relevamientos muy variados en relación a los talleres, el desafío que queda pendiente es presentar, analizar y proponer experiencias de aula/taller en otras disciplinas como las ciencias exactas y las naturales.

Finalmente, si la escuela parte de la transmisión de la cultura hegemónica, aunque amplíe los niveles estandarizados de lo que se denomina "imposición cultural", poco podrá hacer para el despliegue y afirmación de la identidad de los sujetos alumnos que quedan fuera de esa cultura hegemónica. En cambio, una escuela inclusiva que, para su proyecto educativo, parta de tener en cuenta el espacio sociohistórico de pertenencia y la realidad concreta en donde está inserta, y haga de estas circunstancias materia para construir su proyecto curricular, realizará un viraje hacia las propuestas que contemplan el entorno como factor determinante de las prácticas.

Los sujetos se implican transformando la realidad, reflexionando sobre sus acciones, modificándose en esta relación y produciendo nuevos análisis, preguntas y reflexiones teóricas. Éstas, a su vez, vuelven a tensarse en la experiencia, y así se genera, sucesivamente, el avance cognitivo junto al devenir histórico y social del pensamiento humano.

Quizá partiendo desde ahí, como ya se dijo, la escuela sea capaz de modificar y resignificar el lugar de la transmisión, para que no consista en la mera promoción de cánones culturales externos a los sectores populares. En este sentido, la educación popular dentro de las escuelas públicas puede contribuir con el rescate de los valores de la solidaridad y cooperación mutua que permitan pensar un nuevo mañana frente a un destino que aparece como inexorable. 
Revista de la Escuela de Ciencias de la Educación, año 12, nRo. 11, vol. 1, enero a Junio de 2016. Páginas 99-118. ISSN 1851-6297. ISSN EN LINEA 2362-3349. IDENTIDAD Y EMANCIPACIÓN EN EL ÁMBITO DE LA EDUCACIÓN FORMAL. ABORDAJE

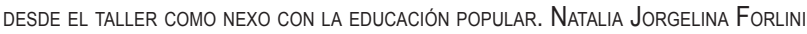

\section{Notas bibliográficas}

(1) Esta elaboración y síntesis conceptual acerca de la identidad es retomada de un escrito anterior de nuestra autoría. Para una mirada más amplia al respecto puede consultarse: Forlini, N. (2010) La configuración de la identidad de los sectores populares en la era de la globalización. Apuntes para pensar las prácticas escolares, en Revista de la Escuela de Ciencias de la Educación. Año 6, N5, (pp. 175 - 189). ISSN 1851-6297. Rosario: Labore Editor.

(2) En el libro El aprendizaje un encuentro de sentidos Silvia Schlemenson (1999) trabaja acerca de la vinculación entre el docente, el niño y el grupo de pares desde la perspectiva psicoanalítica. Aquí pretendemos realizar un enfoque político-pedagógico.

(3) Se toman los aportes del corpus teórico de Pichon-Rivière. Sus aportes conceptuales ayudan a sostener la teoría del aprendizaje que se produce en las dinámicas de taller en educación popular. En este artículo solo se hace mención a ella como marco teórico desde el cual nos posicionamos. En próximos trabajos de escritura se presentará la articulación y examen de cómo se visualizan sus aportes y resultados en la práctica.

(4) El material de campo producido y registrado como observaciones de la implementación de talleres de educación popular en el marco del proyecto de investigación mencionado al inicio, será material de análisis futuro. Se pretende en este escrito, solo problematizar los conceptos en relación a los primeros acercamientos a la experiencia.

\section{Referencias Bibliográficas}

- $\quad$ Aulagnier, P. (1997). La violencia de la interpretación. Del pictograma al enunciado. Argentina: Amorrortu.

- Bleger, J. (1977). Temas de Psicología. (Entrevista y grupos). Buenos Aires: Nueva Visión.

- $\quad$ Elias, N. (1969/1996). La sociedad cortesana. México: Fondo de Cultura Económica.

- Forlini, N. (2010). La configuración de la identidad de los sectores populares en la era de la globalización. Apuntes para pensar las prácticas escolares. Revista de la Escuela de Ciencias de la Educación, Año 6, N5, (pp. 175-189). ISSN 1851-6297. Rosario: Laborde Editor.

- $\quad$ Foucault, M. (1997). Las palabras y las cosas. Madrid: Siglo XXI.

- Franco, Y.; Freire, H.; Loreti, M. (2007). Insignificancia y autonomía. Debates a partir de Cornelius Castoriadis. Buenos Aires: Biblos.

- Freire, P.; Quiroga, A. (1986). El proceso educativo según Paulo Freire y Enrique PichonRivière. Buenos Aires: Cinco.

- $\quad$ Freire, P. (1986). La educación como práctica de la libertad. Bogotá: Siglo XXI.

- - (1994). Cartas a quien pretende enseñar. México: Siglo XXI.

- Meyer, J. Ramírez, F. (2002). La institucionalización mundial de la educación, en Schriewer, J. (comp.) Formación del discurso en la educación comparada. (pp 91-111). Barcelona: Pomares.

- Quiroga, A. Racedo, J. (2007). Crítica de la vida cotidiana. Buenos Aires: Cinco.

- Quiroga, A. (2012). Matrices de aprendizaje. Constitución del sujeto en el proceso de conocimiento. Buenos Aires: Ediciones Cinco.

- Quiroga, A. (2005). Crisis, Procesos sociales, sujeto y grupo. Desarrollos en Psicología Social a partir del pensamiento de Enrique Pichon - Rivière. Buenos Aires: Ediciones Cinco. 
Revista de la Escuela de Ciencias de la Educación, año 12, nRo. 11, vol. 1, enero a Junio de 2016. Páginas 99-118. ISSN 1851-6297. ISSN EN LINEA 2362-3349. IDENTIDAD Y EMANCIPACIÓN EN EL ÁMBITO DE LA EDUCACIÓN FORMAL. ABORDAJE DESDE EL TALLER COMO NEXO CON LA EDUCACIÓN POPULAR. NATALIA JORgELINA FoRLINI

- $\quad$ (1996). Identidad y realidad social, en Revista Temas de psicología social. Año 17, N 15. Buenos Aires: Cinco.

- Racedo, J. [et al] (2004). Patrimonio cultural e identidad. Culturas populares, memoria social y educación. Buenos Aires: Cinco.

- $\quad$ Schlemenson, S. (1996). El aprendizaje, un encuentro de sentidos. Buenos Aires: Kapelusz. 